\title{
¿A qué llamamos salud y enfermedad mental en pediatría? Una mirada reflexiva sobre los diagnósticos psiquiátricos y su relación con nuevas teorías de subjetivación en el niño
}

\author{
What do we call health and mental disorders in pediatrics? \\ A reflexive outlook on the psychiatry diagnosis and its relation \\ with the new subjetive theories in children
}

Dra. Irene Kremer ${ }^{a}$

\section{RESUMEN}

Se discuten las bases conceptuales sobre las que asienta la psiquiatría infanto-juvenil considerando las claras divergencias que se plantean al definir salud y enfermedad mental. Se destaca que la mente no es simplemente un fenómeno biológico y, por ello, son necesarios parámetros evaluativos que incluyan los estados subjetivos, así como pasar de pensamientos lineales a formas complejas que incorporen el valor de la experiencia, del azar y del acontecimiento, entre otras ideas, lo cual posibilitará un cambio de perspectiva: el reconocimiento de indicadores positivos de salud mental y no solo ausencia de síntomas o trastornos. Palabras clave: psiquiatría infanto-juvenil, indicadores de salud mental.

\section{ABSTRACT}

Conceptual basis for Infant and Juvenile Psychiatry are discussed taking into account the clear differences in the definition of Health and Mental Illness. It is emphasized that the mind is not simply a biological phenomenon and, thus, there is a need for assessment parameters that include subjective states, as well as a shift from lineal thoughts to complex forms that include the value of experience, fate, and event, amongst other ideas. This allows a change of perspective: the acknowledgement of the Positive Indicators of Mental Health and not only the absence of symptoms or disorders.

a. Instituto Privado Kremer.

Neuropsiquiatría y Medicina avanzada. Universidad Católica de Córdoba, Facultad de Medicina, Carrera y Post Grado de Psiquiatría Infanto Juvenil.

\section{Correspondencia:}

Dra. Irene Kremer: irene.kremer@ institutokremer.com.ar

Conflicto de intereses: Ninguno que declarar.

Recibido: 9-5-11 Aceptado: 11-8-11
Key words: psychiatry infant and juvenile, indicators of mental health.

doi:10.5546/aap.2011.412

\section{¿A QUÉ LLAMAMOS \\ SALUD MENTAL? ¿QUÉ CONSIDERAMOS ENFERMEDAD EN PSIQUIATRÍA Y PSICOANÁLISIS DE NIÑOS Y ADOLESCENTES?}

\section{Cómo definir salud y enfermedad mental}

La psiquiatría infanto-juvenil es, por definición, una especialidad que se ocupa de la salud y de la patología mental de los bebés, niños y adolescentes. Pero, ¿a qué llamamos salud mental? ¿Y qué consideramos enfermedad en psiquiatría infanto juvenil (PIJ)?

Aun a riesgo de ocuparnos de temas aparentemente obvios, formulamos estas preguntas a fin de acercarnos a la comprensión de los conceptos fundamentales sobre los que se desarrolla dicha especialidad.

Coincidimos con Winnicott ${ }^{1}$ cuando dice que: "En los asuntos humanos nada es incuestionablemente definido y ¿quién puede decir dónde termina la salud $y$ dónde comienza la enfermedad?". Este pensador, pediatra y psicoanalista, nos recuerda también que "Salud no es comodidad. Los temores, los sentimientos conflictivos, las dudas y las frustraciones son tan característicos en la vida de una persona sana como los rasgos positivos. Lo importante es que esa persona sienta que está viviendo su propia vida y asumiendo la responsabilidad de sus actos y omisiones y sea capaz de atribuirse el mérito cuando triunfa y la culpa cuando fracasa."

Divergencias conceptuales en las definiciones de salud y enfermedad mental en relación de una con la otra

El diagnóstico de enfermedades mentales es diferente al consenso logrado en las enfermedades físicas, las que se definen contra estándares de funcionamiento normal, que provienen de grados de presión arterial, pulso, agudeza visual y parámetros de laboratorio, entre otros. En psiquiatría, debemos considerar simultáneamente, al menos dos aspectos. Primero, 
aceptar que hay ciertos acuerdos consensuados que ayudan a brindar confiabilidad a los efectos prácticos del médico que trata a un paciente y para los investigadores que definen diagnósticos. En segundo lugar, reconocer ciertas características esenciales que no es posible resignar, aun si no es posible definirlas con total precisión: aquello que hace a cada persona única, en particular cuando se trabaja con niños y adolescentes.

En PIJ, quizás más que en cualquier otra especialidad, debemos poder tolerar, como el aviador con su principito, ${ }^{2}$ muchos momentos en los que no entendemos totalmente quién es ese sujeto que está frente a nosotros, qué busca, qué le impide encontrar lo que necesita o desea. Y nuestra función es, muchas veces, ser como un medio facilitador para que cierto funcionamiento mental pueda a veces inaugurarse y otras volver a funcionar, permitiendo que emerjan dichas respuestas.

Como médicos (pediatras, psiquiatras y psicoanalistas), es útil recordar que la enfermedad no es solo la patología en sí misma, sino y sobre todo, la experiencia que tiene el paciente de su enfermedad. Y destacar que dicha experiencia se va construyendo en los encuentros cotidianos del niño no solo con sus padres, también con su pediatra, con sus maestros y, por supuesto, con su terapeuta, y que mientras todo ello ocurre se van gestando los sentimientos de identidad, autoestima, confianza y de todo aquello que conforma la personalidad.

De modo que un niño/a podrá ir pensando sobre sí mismo: "Soy un celíaco, soy un Trastorno por Déficit Atencional, soy un superdotado o el más tonto de todos, soy débil y no puedo tolerar ningún dolor físico ni ninguna frustración o, por el contrario, soy invulnerable a todas las agresiones, etc., etc.". O podrá ir construyendo una idea de sí mismo en la que él es Juan o Sofía, una persona a la que le pasan cosas, cuyo cuerpo está involucrado, también sus emociones, sus impulsos, sus pensamientos, pero su identidad no está dada por una única situación que lo define, a veces durante toda la vida.

Por otra parte, si solo aceptamos parámetros evaluativos que puedan ser probados objetivamente y excluimos la subjetividad y las hipótesis que la involucran, negándoles cualquier validez científica, estamos en peligro de caer en lo que algunos llaman la "psiquiatría sin sentido": la disciplina perdería relevancia respecto de lo que experimentan los pacientes y para lo cual buscan ayuda; sus estados subjetivos.

Es bien conocido que la psiquiatría clásica, amparada en cierta forma de usar el DSM, la CIE-10 y otros manuales diagnósticos, refleja un marco de trabajo en el que no hay espacio para la psique excepto en la forma de enfermar. De modo que, en cierto imaginario social, consultar a un psiquiatra solo puede ofrecer como resultado la confirmación de un trastorno: "Dígame, doctor: ¿El loco soy yo o es él?"

Tampoco desde el psicoanálisis hay parámetros positivos para definir lo saludable, salvo por la tendencia a atribuirle cierta afinidad con los procesos de funcionamiento neuróticos. Pareciera que muchas veces se olvida el tremendo sufrimiento o inhibición que conlleva, problemática que fue revisada en profundidad por Rodulfo, ${ }^{3}$ entre otros autores.

Un cambio de perspectiva es absolutamente necesario para que los avances en la comprensión del funcionamiento mental sean incorporados en los consultorios que se ocupan de niños y adolescentes brindando valiosas herramientas, que no solo contribuirán a ayudar a los pacientes y sus familias, sino que también enriquecerán la experiencia profesional.

\section{REVISIÓN DE ALGUNOS CONCEPTOS FUNDAMENTALES}

Constitución subjetiva temprana

En los últimos años se viene produciendo una gran revolución en los paradigmas desde los cuales miramos, escuchamos y, sobre todo, pensamos nuestra especialidad. Las primeras teorías psicoanalíticas analizaban el desarrollo psicoemocional desde la reconstrucción de relatos de pacientes adultos con patologías diversas, sin detenerse en observaciones del niño sano (teorías adultomórficas y pato-céntricas). ${ }^{4-5}$ Por otra parte, las corrientes conductistas y cognitivistas dedicaron sus investigaciones a recoger datos sobre cómo y cuándo los niños iban adquiriendo destrezas, habilidades y conocimientos, pero dejaban fuera el intento de acceder a la cualidad de vivencia subjetiva que el bebé y el niño pequeño experimentaban a medida que iban desarrollando dichas capacidades. La orientación actual se dirige cada vez más a ampliar, depurar e integrar ambas bases de información, ya que toma en cuenta los hallazgos de la observación experimental y, sobre la base de los conocimientos obtenidos con adultos, intenta inferir las experiencias subjetivas especificas de los bebés y niños.

\section{Indefensión/aptitudes preconstituidas/ juego y creatividad/cultura}

Stern ${ }^{5}$ y Lebovicci, ${ }^{6}$ entre otros, demuestran que, junto a la innegable indefensión del recién 
nacido, la cría humana trae consigo aptitudes preconstituidas para relacionarse con el medio, aptitudes que son propias de la especie pero que, además, hacen a la singularidad de cada sujeto, ya observables desde el nacimiento. Por ejemplo, se puede afirmar que el bebé no tiene una disposición pasiva ni de aislamiento (autismo) con su entorno. Muy por el contrario, se destaca la prevalencia de los períodos de calma con una disposición activa al descubrimiento del mundo humano y al mundo en general, en donde la alegría, y no la angustia, es el afecto que más a menudo acompaña a dichos descubrimientos. ${ }^{7}$ Por otra parte, la menor dotación instintiva de los seres humanos en relación a otras especies ya no son pensadas solo como una desventaja por la indefensión a la que expone a su cría, sino que, al requerir un mayor período de protección y amparo (profundamente estudiado en la Teoría del Apego) 4 "abre el juego" a la creatividad, a la inventiva, a la expresión simbólica y cultural en sus más diversas expresiones.

\section{La enfermedad mental no es simplemente un fenómeno biológico}

La mente es dependiente del cerebro pero no es reductible a él, de modo que esta dependencia no es identidad. Algo extra ocurre cuando el estado cerebral conduce a un estado mental; el estado mental "toma lugar" o "surge de" la constelación de los estados cerebrales que lo subyacen. Diferentes niveles interactúan unos con otros configurando sistemas complejos, que son vistos como un todo, más que analizados como sus partes, funcionando separadamente, dotando a la vida mental de sus propias leyes.

Gahemi ${ }^{8}$ explicita claramente estos interrogantes: "¿Sabemos qué es dolor si entendemos todo sobre la neurobiología de éste, pero nunca lo hemos experimentado? ¿Es lo mismo la neurobiología del dolor que el dolor en sí?... En otras palabras, y volviendo a un lenguaje filosófico más antiguo, ¿hay algo subjetivo acerca de las experiencias mentales que sea diferente de las bases objetivas de estas experiencias? Y, ies este aspecto subjetivo inefable? Es decir, ¿es ello por definición incomprensible de una manera científica objetiva? o ¿hay alguna versión del conocimiento subjetivo que sea accesible?"

\section{Dicotomía entre los enfoques biológico y psicosocial. Lo simple y lo complejo}

Durante mucho tiempo ha existido en psiquiatría la separación entre una aproximación con predominancia psicológica y otra con prevalencia biológica. En parte debido a que, como lo explicita Horstein9 "Hasta hace pocas décadas predominó en la ciencia la aspiración a la simplicidad. Lo simple estaba ahi, apenas oculto por las apariencias cambiantes. Es simple y puede ser aislado; aislamiento que permite a los especialistas ser expertos en compartimientos y cooperar con eficacia en sectores de conocimiento no complejos. Pero ese paradigma implica una lógica que extiende sobre la sociedad y las relaciones humanas restricciones y funciones propias de la máquina artificial y de la visión determinista y mecanicista que la máquina origina..." "Hoy la ciencia aborda lo complejo, se abre a lo imprevisible... Un bucle auto-organizador reemplaza la linealidad causa-efecto por la recursividad. Recursivos son los procesos en los que los productos son al mismo tiempo productores de aquello que los produce".

\section{Implicancias clínicas del abordaje a la concepción de complejidad}

Estos conceptos atañen a cuestiones fundamentales que hasta hace poco eran abordadas, mayoritariamente, como pares opositivos: genética o crianza; padres o hijos; vivencias traumáticas o vulnerabilidad constitucional; psicoterapia o tratamiento farmacológico. Considerar la complejidad nos exime de optar por una u otra opción de manera categórica y nos conduce más bien a considerar los distintos efectos que cada componente ejerce sobre los demás. Tanto en salud como en patología.

Dio Bleichmar ${ }^{4}$ enfatizó estos puntos al afirmar que: "La comprensión de los fenómenos del desarrollo requiere conceptos relacionales o co-activos de la causalidad en oposición a causas únicas que operan en un supuesto aislamiento...

Cuando se habla de co-acción como el corazón de la causalidad evolutiva, lo que se quiere enfatizar es que necesitamos especificar alguna relación entre al menos dos componentes del sistema en desarrollo (gen-gen, persona-persona, organismo-organismo, organismoambiente, actividad-conducta motora). El concepto usado más frecuentemente para designar la co-acción es el relativo a la experiencia".

\section{El valor de la experiencia}

Los psicoanalistas que trabajamos con niños, los pediatras y los psiquiatras infantiles tenemos el privilegio de estar presentes en múltiples situaciones en las que el psique-soma aún no se ha diferenciado, en momentos en los que la mente está emergiendo y en los que el bebé está empezando a configurar una perspectiva del mundo y de sí mismo. Dichas experiencias contribuirán a 
la humanización solo si se "sostiene" al niño y a los involucrados en su crianza cada vez que lo necesiten, y desde todas estas disciplinas podemos colaborar a que ello ocurra. Winnicott ${ }^{1}$ lo expresa como sigue: "Incluyo el uso de experiencias inherentes a la existencia, tales como la terminación (y por ende la no terminación) de procesos que, vistos desde fuera, acaso parezcan puramente fisiológicos, pero que corresponden a la psicología de la criatura y tienen lugar dentro de un complejo campo psicológico y que son determinados por la conciencia y la identificación de la madre".

Una investigación experimental reciente ${ }^{10}$ permite resaltar el valor de la experiencia; en ella, se demuestra que las exposiciones prolongadas a la música clásica (en particular la sonata K448 de Mozart) en los niños de 3-4 años conducían a una mejora del $30 \%$ en los tests de razonamiento espacial y temporal, comparados con un grupo que había recibido clases de informática durante seis meses.

El funcionamiento de la música de Mozart no se entiende mucho, pero es una clara ilustración de cómo una forma específica concreta de experiencia subjetiva (en este caso la familiaridad con las formas musicales) puede interactuar al nivel de funcionamiento cerebral con una capacidad psicológica importante, aparentemente independiente.

\section{Un lugar para el azar y el acontecimiento}

Es habitual escuchar que todo lo importante que sucede en la vida de una persona está determinado en los primeros años de vida y que, para bien o para mal, ello no podrá ser modificado sustancialmente a posteriori. Sin embargo, en la actualidad, las diferentes teorías consideran cada vez más la posibilidad de incorporar lo desconocido, lo inédito, lo especial que pueda suceder y a considerar cómo cada uno metaboliza dichos acontecimientos. Se rescata el azar como factor interviniente y el valor de una re-escritura, no de copia ni fotocopia de lo vivido ni de lo trasmitido por los padres: reescritura de los mitos familiares, de la disponibilidad o carga genética, de las capacidades innatas. ${ }^{10-11}$

\section{Indicadores positivos de salud mental en niños y adolescentes}

Comienzan a emerger distintos indicadores, que nos facilitan detectar aspectos saludables en el desarrollo emocional de un niño, más allá de la eventual presencia de síntomas o trastornos y que, por lo tanto, amplían y enriquecen la pers- pectiva desde donde miramos, escuchamos y, sobre todo, pensamos a nuestros pacientes.

La semiología de la salud mental indaga en la capacidad de ser espontáneo y de tener iniciativas, así como la capacidad para estar con otros y para permanecer a solas. Importa saber si el niño puede ir reconociendo sus deseos e implementar las acciones necesarias para satisfacerlos; si está aprendiendo a descubrir la subjetividad de los otros y a respetarla; si puede sentir culpa y responsabilidad por sus actos, tanto como pena o dolor ante una pérdida; si siente necesidad de explorar el mundo que lo rodea y confianza por personas de su entorno. Nos interesa saber si tiene imaginación, si puede soñar, cómo se relaciona con su cuerpo y qué fantasías se tejen en relación a su funcionamiento corporal. Debemos explorar si puede pedir ayuda cuando la necesita y cómo se las arregla para tolerar las pequeñas o graves frustraciones cotidianas.

$\mathrm{Al}$ indagar todas estas capacidades trans-nosográficamente, encontramos que no hay dos niños con trastorno por déficit de atención, con neurosis o psicosis infantiles que sean iguales. Y parte esencial de nuestro trabajo es descubrir y cuidar la singularidad de nuestros pacientes, al tiempo que ayudamos a superar sus dificultades.

\section{Una revalidación de la importancia clínica de una semiología de la salud}

Se pretende reconocer la salud por indicadores clínicos positivos y no solo por la ausencia de síntomas. Considerando que "salud no es comodidad" podemos afirmar que cuando todo esté demasiado bien y el niño/a nunca se angustie, se ponga triste o se enoje, sus padres siempre interpreten correctamente y en el momento oportuno lo que necesita, no se presenten conflictos o estos se "solucionen de inmediato", será necesario explorar las capacidades reales de las que dispone ese niño y su entorno cercano para crecer emocionalmente y sentirse vivo -algo más que estar con vida-, tanto como si presenta síntomas manifiestos.

En una consideración global de lo arriba expresado, aplicable a amplios campos del trabajo médico, psiquiátrico y psicoanalítico, se pasa de una visión reduccionista a otra de carácter integrador y pluralista. En otras palabras, esta concepción implica un prospectivo y enorme esfuerzo educativo que incorpore información crítica selectiva para el nivel profesional así como actividades docentes y educativas a nivel general de los equipos de salud y en particular en pediatría y salud mental. 


\section{BIBLIOGRAFÍA}

1. Winnicott D. El concepto de individuo sano. [Acceso: 18 de abril 2011]. Disponible en: http://psicoanalisis.org/ winnicott/.

2. De Saint-Exupery A. El Principito. Buenos Aires: Ed. Emecé. 2005.

3. Rodulfo R. Las neurosis y las formaciones de lo transicional. En: La problemática del síntoma. Buenos Aires: Ed. Paidós. 1997; Págs. 43-86.

4. Dio Bleichmar E. La compleja unidad de la experiencia temprana. En: Manual de psicoterapia de la relación padres e hijos. Barcelona: Ed. Paidós Ibérica. 2005; Págs. 15-22.

5. Stern D. Los interrogantes y su trasfondo. Perspectivas y enfoques de la infancia. En: El mundo interpersonal del infante. Buenos Aires: Ed. Paidós. 1985; Págs. 17-29.

6. Lebovici S. El lactante, su madre y el psicoanalista. En: Las interacciones precoces. Buenos Aires: Amorrortu editores. 1983; Págs. 89-130.
7. Rodulfo R. Alegría y angustia, angustia y alegría. Rondó de un descuido. En: Futuro Porvenir. Buenos Aires, México: Ed. Novedades Educativas. 2008; Págs. 69-82.

8. Ghaemi N. Psiquiatría: conceptos. The Johns Hopkins Univesity Press Ed Español Santiago de Chile: Ed. Mediterráneo. 2008.

9. Horstein L. Intersubjetividad y práctica psicoanalítica. Desafíos actuales. En: Inter-subjetividad y Clínica. Buenos Aires: Ed. Paidós. 2003; Págs. 201-211.

10. Fonagy P. Genética, psicopatología evolutiva y teoría psicoanalítica: el argumento para terminar con nuestro (no tan) espléndido aislamiento. Psychoanalytic Inquiry 2003: 23 (2). [Acceso 4 de abril 2011]. Disponible en www.aperturas.org/revistas.

11. Rodulfo R. Serie y Suplemento. En: El psicoanálisis de nuevo. Buenos Aires: Ed. Eudeba. 2004; Págs. 21-36.

“Un escritor, un periodista, un intelectual, sólo puede escribir, alertar, hablar. Pero es necesario que del otro lado esté el que lea, escuche y se haga eco. Se precisa del interlocutor sensible, y es lo que falta. El Estado está ausente y la sociedad, cada vez más entregada a la estupidez. Los políticos se ocupan de lo inmediato y redituable, y los niños no votan". 